\title{
Des pratiques éducatives fondées sur les opinions aux pratiques éducatives fondées sur les preuves
}

\author{
From opinion-based to evidence-based education
}

De par le monde, les dix dernières années ont été marquées par des mouvements importants quant à la manière de penser la formation des professionnels de la santé. Ainsi, le déploiement de l'approche par compétences inspire de nombreuses réformes dans la plupart des pays. C'est le cas, par exemple, au Canada où le cadre de référence CanMEDS oriente de façon très prescriptive les différents programmes de formation médicale initiale. C'est aussi le cas en Suisse où le référentiel PROFILES (Principal Relevant Objectives and Framework for Integrative Learning and Education in Switzerland), lui-même inspiré de CanMEDS, vient de se substituer à l'ancien catalogue SCLO (Swiss Catalogue of Learning Objectives) pour définir les résultats attendus (acquis d'apprentissage) à l'issue du programme prégradué sous forme de compétences. En France, les mêmes orientations ont conduit à implanter l'approche par compétences dans la formation infirmière en 2009 et sous-tendent le déploiement des réformes des premier et deuxième cycles des études de médecine, planifié pour la prochaine rentrée universitaire.

Ces réformes sont autant d'opportunités pour chaque enseignant et chaque formateur de se pencher sur ses pratiques, en se posant la question suivante : «Ma façon de former et d'évaluer les étudiants et/ou les professionnels de santé est-elle conforme aux règles de bonnes pratiques pédagogiques, issues des données de la recherche?»

La question pourrait paraître incongrue lorsqu'elle s'adresse à des professionnels de santé dont personne - en premier lieu les patients - ne pourrait aujourd'hui imaginer que leurs pratiques des soins s'éloignent des recommandations scientifiques. Pourtant, force est de constater que, malgré l'appel, lancé il y a une vingtaine d'années, visant à encourager le passage d'une éducation fondée sur les opinions à une éducation fondée sur les preuves [1], nous continuons souvent actuellement à former comme nous soignions il y a un demi-siècle, en dehors de toute logique pédagogique et rationalité scientifique. Les étudiants se montrent d'ailleurs étonnamment compréhensifs, voire complices de la situation.

De nombreux facteurs peuvent être évoqués afin d'expliquer la situation. Nous en retiendrons quatre.

En premier lieu, il convient d'admettre que, si de bonnes pratiques soignantes produisent généralement des effets immédiats, ou du moins, à court terme, générant ainsi de la satisfaction chez le soigné et le soignant, renforçant par conséquent les pratiques de ce dernier, il n'en est pas toujours de même des pratiques efficaces de formation. De fait, il est souvent difficile de se rendre compte que l'on a bien formé un étudiant ou un groupe d'étudiants, d'autant plus que si le dispositif éducatif souffre d'un manque d'alignement pédagogique, il est même possible que les résultats académiques d'étudiants bien formés soient négativement affectés [2]. C'est par exemple ce qui s'est produit lorsque certaines facultés, notamment françaises, ont essayé d'implanter dans leur cursus l'apprentissage par problèmes (APP). La qualité des apprentissages a très certainement été améliorée sur le plan du développement des compétences professionnelles des étudiants, mais les institutions ont continué à évaluer la quantité de connaissances retenues par les intéressés et non leurs capacités à utiliser ces connaissances pour résoudre les problèmes des patients. Cette situation a conduit à une altération des performances aux examens, dans la mesure où ces derniers ne cherchaient pas (ou peu) à documenter les connaissances que les étudiants étaient supposés avoir acquises grâce à l'APP (notamment, les connaissances d'action).

Dans un deuxième temps, nous souhaiterions souligner que chacun a un avis personnel et généralement très tranché sur l'éducation [3]. Qui n'a jamais assisté à une réunion de parents d'élèves, une sortie entre amis ou un repas de famille, où les participants s'invectivaient à coup de «C'est n'importe quoi cette réforme!», «Ce prof est vraiment nul et ne sait pas faire cours, on se demande bien ce qu'il fait là !», ou encore «C'est scandaleux de poser des questions pareilles à un examen!». Les mécontents étaient-ils dans le vrai? Probablement pas. Pour une raison simple : leur jugement relève quasiment toujours de constats empiriques, construits au gré de leurs expériences pédagogiques [3].

Il s'agit là du troisième facteur que nous souhaitions exposer. L'essentiel de nos expériences pédagogiques correspond à notre vécu en tant qu'élève, puis étudiant. Dès lors que nous avons été soumis à un modèle d'enseignement pendant plus de 20 ans, il est compréhensible que lorsque nous endossons la fonction de formateur ou d'enseignant, nous reproduisions ce modèle à l'identique. Modèle qui ne doit d'ailleurs pas si mal marcher, 
puisque nous en avons bénéficié et sommes devenus de bons professionnels!

Nous voici rendus au quatrième facteur, qui constitue probablement le principal obstacle au changement, au regard de notre fonctionnement cognitif: pourquoi diable ferions-nous l'effort de modifier notre façon de faire, alors que personne n'en ressent véritablement le besoin? Ni les étudiants (quoi de plus confortable que d'être assis au fond d'un amphithéâtre ou d'une salle de cours, de noter ce que dit l'enseignant ou le formateur, et de restituer un contenu scientifique en cochant les cases d'une QCM...), ni les enseignants (quoi de plus commode que de réciter son cours ou de demander à un stagiaire de regarder faire un expert et de reproduire ce qu'il observe...), ni l'institution (quoi de plus économique que le paradigme d'enseignement, que nous venons de décrire...), ni même les politiques (quoi de plus parlant que nos excellents indicateurs de santé qui prouvent que... l'accès aux soins est en France extraordinaire et bénéficie à tout le monde). Et ce ne sont pas quelques pédagogues, enseignants «atypiques» et étudiants rêveurs, parfois pénibles, souvent incompréhensibles et toujours idéalistes, qui vont nous empêcher de tourner en rond.

Maintenant que nous avons identifié les (fortes) résistances au changement, le moment est venu de prendre conscience que quelque chose ne va pas. Nous nous contenterons pour cela de citer deux chiffres. Dix à quinze pour cent des étudiants connaîtront des difficultés au cours de leur formation [4-6]. Ces difficultés concernent fréquemment leur capacité à résoudre des tâches cliniques en mobilisant un raisonnement adapté [7]. Rappelons ici que la résolution de tâches est l'activité principale du soignant et qu'il y consacre l'essentiel de ses journées. Deuxième chiffre: selon les spécialités, jusqu'à un diagnostic sur six posé par un médecin est erroné (et il n'y a pas vraiment de raison pour que la situation soit différente chez les autres professionnels de santé) [8-10]. Ces chiffres sont-ils acceptables? Si l'on se place dans une perspective de responsabilité sociale des facultés et des écoles de santé, en considérant notamment le point de vue du patient [11], la réponse s'impose d'elle-même.

Les réformes des études de santé sont autant d'opportunités pour inscrire nos pratiques dans le respect des recommandations scientifiques et de l'éducation fondée sur les preuves. Il conviendra cependant de ne pas tomber dans les travers parfois constatés de la médecine factuelle, qui, de façon souvent réductrice et dans le cadre d'une confusion, marquée par l'hégémonie positiviste, entre la science médicale et la pratique médicale, ont conduit certains décideurs et professionnels de santé à penser que pour bien soigner, il suffisait d'appliquer des recommandations d'experts ou des procédures standardisées, transformant par là même les soignants en techniciens du soin. Les pratiques soignantes, tout comme les pratiques éducatives, doivent rester des pratiques personnalisées, éclairées par les données de la science et instrumentées par des techniques, afin de pouvoir répondre aux besoins idiosyncrasiques de chaque patient et de chaque étudiant.
La capacité à cheminer de pratiques éducatives fondées sur les opinions - dont on comprendra, sur la base des précédents paragraphes, qu'elles s'installent naturellement et, le plus souvent, indépendamment de la volonté de ceux qui les adoptent - vers des pratiques fondées sur les preuves pourrait constituer un attribut du professionnalisme et de l'identité de tout formateur ou enseignant en sciences de la santé, dont, au final, tout le monde pourrait bénéficier: les étudiants (qui apprendront mieux et de façon plus engagée), les enseignants (qui s'ennuieront moins dans leurs activités d'enseignement et enseigneront comme ils soignent, retrouvant ainsi une cohérence utile à leur bien-être psychique), l'institution (qui remplira sa mission première de responsabilité sociale) et les politiques (qui pourront enfin communiquer sur l'excellence de notre système de formation des professionnels de la santé).

Thierry PELACCIA ${ }^{1,2, *}$

${ }^{1}$ Directeur du Centre de formation et de recherche en pédagogie des sciences de la santé (CFRPS),

Faculté de médecine de Strasbourg, Strasbourg, France

${ }^{2}$ Secrétaire général de la Société internationale francophone d'éducation médicale (SIFEM)

* Mailto: pelaccia@unistra.fr

\section{Références}

1. Harden RM, Grant J, Buckley G, Hart IR. BEME guide No. 1: best evidence medical education. Med Teach 1999:21:553-62.

2. Bertrand C, Dory V, Pelaccia T, Charlin B, Hodges B. Comprendre les principes généraux de l'évaluation. In: Pelaccia T (Ed.). Comment [mieux] former et évaluer les étudiants en médecine et en sciences de la santé? Louvainla-Neuve: De Boeck Supérieur, 2016:343-55.

3. Niculescu RM, Norel M. Human resources as leading and supporting actors of a curriculum reform. Procedia Soc Behav Sci 2013;93:432-6.

4. Yates J, James D. Predicting the "strugglers": a case-control study of students at Nottingham University Medical School. BMJ 2006;332:1009-13.

5. Faustinella F, Orlando PR, Colletti LA, Juneja HS, Perkowski LC. Remediation strategies and students' clinical performance. Med Teach 2004;26:664-5.

6. Smith CS, Stevens NG, Servis M. A general framework for approaching residents in difficulty. Fam Med 2007;39: 331-6.

7. Audétat MC, Caire Fon N. Aider les étudiants en difficulté. In: Pelaccia T (Ed.). Comment [mieux] superviser les étudiants en sciences de la santé dans leurs stages et dans leurs activités de recherche. Louvain-la-Neuve: De Boeck Supérieur, 2018:161-83.

8. Berner E, Graber M. Overconfidence as a cause of diagnostic error in medicine. Am J Med 2008;121:S2e23.

9. Graber M, Gordon R, Franklin N. Reducing diagnostic errors in medicine: what's the goal? Acad Med 2002;77: 981-92.

10. Kuhn GJ. Diagnostic errors. Acad Emerg Med 2002;9: 740-50.

11. Pestiaux D. La responsabilité sociale des institutions de formations en santé. Pédagogie Médicale 2015;16:163-5. 